\title{
A New Ache in The World of Joints
}

\author{
Jahan Marcu ${ }^{1}$, Teresa A Simon ${ }^{2 *}$, Phil Molloy ${ }^{3}$ \\ ${ }^{1}$ Marcu Enterprises USA, Marcu and Arora and Physicians Research Center, LLC USA \\ ${ }^{2 *}$ Physicians Research Center, LLC USA, Adjunct Fellow Univ of PA, Center for Public Health Initiatives, USA \\ ${ }^{3}$ Rheumatology Private Practice, MA, USA
}

*Corresponding author: Teresa A Simon, Physicians Research Center, LLC USA, Adjunct Fellow Univ of PA, Center for Public Health Initiatives, USA. prctrials@gmail.com

\author{
Received Date: July 09, 2021 \\ Published Date: August 31, 2021
}

\section{The State of the Joint}

Patients with rheumatic diseases are afflicted with many different symptoms depending on the disease, however the one symptom that is common among all the rheumatic diseases is pain. In an explorative effort to combat this pain since currently available treatments frequently provide insufficient relief, this patient population has been increasing use of cannabis and hemp products for symptom management.

This increase is documented in a recent study that evaluated the prevalence of cannabis use in a rheumatic disease population from the FORWARD registry, a registry of rheumatic disease patients [1]. This study documented an increase in cannabis use nationwide (from 6.3\% to 18.4\%) and at the state level regardless of rheumatic disease diagnosis or cannabis legality status between 2014 and 2019 [2]. Results are consistent with the increased prevalence of cannabis use in patients with rheumatic diseases in Canada, (4.3\% in 2016 and $12.6 \%$ in 2020) [3]. Therefore, the prevalence of cannabis use among individuals with rheumatic diseases has tripled in both the Canadian and US population in similar timeframes.

Given this increase, there remains a dearth of evidence on the long-term use and impact of cannabis and/or hemp-based products in the rheumatic disease community. Health care providers consistently hear how patients are reluctant to accept recommendations and prescriptions for proton pump inhibitors, statins, biologic anti-rheumatic therapies because of perceived dangers and toxicities, yet some patients will accept a cannabis or hemp-product as a treatment (e.g., for cancer) as if both its efficacy and safety is more solidly proven [4]. A recent study from the National Survey on Drug Use and Health showed an 18\% relative decrease in perceived risk of cannabis [5].
Simultaneously, there has been an increase in public support for the federal legalization of cannabis in the United States (US) [6]. Presently, most of the United States population lives in a State where an adult can purchase a cannabis product as easily as a beer from a licensed dispensary. There are also several ways to access hemp-products outside of State licensed stores. Hemp products are defined as any cannabis-product with less than $0.3 \% \Delta 9$ tetrahydrocannabinol (THC) by weight. These hemp products are often rich in cannabidiol (CBD) and other plant compounds with significant pharmacological activity, but often skirt much regulatory oversight (e.g., labeling accuracy) beyond the THC limit [7].

Despite the increased availability and consumption of cannabis and hemp products, there is mounting evidence that consumers, the public, and healthcare professionals are confused about how to read the labels of these products, what exactly are in these products, and what potential benefits and side effects they might have [7-10]. An example is from a recent pilot study of consumers and their knowledge and attitudes [11]. The data demonstrate a lack in understanding of cannabis while supporting legalization. There seems to be a disconnect between support in the legalization of cannabis and the understanding of its risks, benefits, and potential adverse effects. Admittedly there is evidence of potential benefit, such as with spasticity, nausea and vomiting associated with chemotherapy, seizures, and pain to mention a few [12]. However, and often overlooked, is the evolving literature documenting potential side effects of cannabis on reproductive health, cardiovascular health, mental health, and sensorimotor skills/reaction times impacting ability to drive safely. Despite the availability of adverse effects data, the balance between risks and 
benefits of cannabis and hemp does not appear to have slowed down public support of cannabis.

Consumers have been going through a trial-and-error process with the use of cannabis products in different states [13]. Pain is among the most common indications associated with the use of medical cannabis [14]. There is some evidence that cannabis is associated with reductions in opioid use across pain disorders [1519]. In addition, cannabis has become increasingly popular as a go to treatment for patients with rheumatic diseases [20].

\section{Education is The Biggest Challenge Facing Patients}

Patients who are considering using these products should be able to receive sound education and speak with qualified individuals that have a certain level of education regarding cannabis and hemp. Who should be responsible for this education on cannabis, hemp, and related products? In lieu of a national consensus on the issue, we can only speculate. Several states have made this the responsibility of a pharmacist, which is required to be available for patient consultations. This approach may work for a niche patient group but cannabinoid containing products can be purchased outside of dispensaries, in places such as gas stations. Are we planning to put pharmacists in every retail outlet that sells cannabis-derived products?

Another viewpoint is that we should rely on the companies making the products. If a company is going to make even an indirect claim about its product, then the company should provide evidence of such a claim which includes the safety data, product standards and labelling. Others suggest it is society's responsibility. Or the government's? Or organized medicine's? Or is it buyer beware? Society has created the incentives for cannabis companies to do what they are setting out to do - make a profit. Whose responsibility is it to ensure education remains unclear. In the end, the education of health care professionals and patients should be one of the most important aspects of national legalization [21]. Is it possible that the members of the cannabis and hemp industry are taking advantage of the public's lack of knowledge? There are many examples of the issues that can arise from a few bad apples in these industries [22]. Most recently the commercial availability of delta-8 THC synthesized from hemp CBD has led to an increased number of cases presenting at poison control centers and reported adverse events to the FDA related to the products containing this compound [11].

\section{Conclusion}

There is a well-recognized public perception that these substances are safe, leading to a possible underappreciation of potential adverse effects. This situation may exist, at least in part, because cannabis is not treated as a "drug" from a regulatory perspective. The public may believe it is safe because they perceive that the regulatory agencies have "signed off" or "passed" on it [9].

A summary from a recent paper: Sarzi-Puttini et al. states "it's necessary to have educational programs that modify the concerns and widespread preconceptions related to cannabis and rheumatic diseases in the medical community by increasing confidence. More extensive basic and clinical research on the mechanisms and clinical utility of cannabis and derivatives in various diseases and their long-term side effects is necessary" [23].

In summary, little is known about the efficacy and safety of medical cannabis or hemp products in the treatment of patients with rheumatic diseases. Despite this dearth of knowledge, or perhaps because of it, education is urgently needed for both patients and health professionals to help them navigate the use of these products while minimizing risks. Presently we must strive to do the best that we can with an incomplete knowledge base, while we wait for desperately needed data from industry and academia to help understand the benefits and mitigate the risks.

\section{Conflict of Interest}

None

\section{Acknowledgements}

The authors are grateful to Dr. Sara Jane Ward and Erin Heaning for their review and input.

\section{References}

1. Wolfe F, Michaud K (2011) The National Data Bank for rheumatic diseases: a multi-registry rheumatic disease data bank. Rheumatology 50(1): 16-24.

2. 2. Wipfler K, Simon TA, Katz P, Wolfe F, Michaud K (2021) Increase in Cannabis Use Among Adults with Rheumatic Diseases: Results from a 2014-2019 US Observational Study. Arthritis Care Res Published online.

3. Ste-Marie PA, Shir Y, Rampakakis E, Sampalis JS, Karellis A, et al. (2016) Survey of herbal cannabis (marijuana) use in rheumatology clinic attenders with a rheumatologist confirmed diagnosis. Pain 157(12): 2792-2797.

4. Abrams DI, Guzman M (2020) Can Cannabis Cure Cancer. Jama Oncol 6(3): 323.

5. Han BH, Funk-White M, Ko R, Al-Rousan T, Palamar JJ (2021) Decreasing perceived risk associated with regular cannabis use among older adults in the United States from 2015 to 2019. J Am Geriatr Soc. Published online 2021.

6. Fataar F, Goodman S, Wadsworth E, Hammond D (2020) Consumer perceptions of 'legal' and 'illegal' cannabis in US states with legal cannabis sales. Addict Behav 112: 106563.

7. Bonn-Miller MO, Loflin MJE, Thomas BF, Marcu JP, Hyke T, et al. (2017) Labeling Accuracy of Cannabidiol Extracts Sold Online. Jama 318(17): 1708-1709.

8. Marcu J (2020) The legalization of cannabinoid products and standardizing cannabis-drug development in the United States: a brief report. Dialogues Clin Neurosci 22(3): 289-293.

9. Kosa KM, Giombi KC, Rains CB, Cates SC (2017) Consumer use and understanding of labelling information on edible marijuana products sold for recreational use in the states of Colorado and Washington. Int J Drug Policy 43: 57-66.

10. Kruger DJ, Korach NJ, Kruger JS (2021) Requirements for Cannabis Product Labeling by U.S. State. Cannabis Cannabinoid Res Published online 2021.

11. Simon TA, Gomez A, Marcu J (2021) What Cannabis related data is Reported in the FDA FAERS Database. In: Cannabis Research Conference 2021.

12. Abrams DI (2018) The therapeutic effects of Cannabis and cannabinoids: An update from the National Academies of Sciences, Engineering and Medicine report. Eur J Intern Med 49: 7-11. 
13. MacCallum CA, Russo EB (2018) Practical considerations in medical cannabis administration and dosing. Eur J Intern Med 49: 12-19.

14. Ataiants J, Fedorova EV, Wong CF, Iverson E, Gold JI, et al. (2021) Pain Profiles among Young Adult Cannabis Users: An Analysis of Antecedent Factors and Distal Outcomes. Subst Use Misuse 56(8): 1144-1154.

15. Abrams DI, Couey P, Shade SB, Kelly ME, Benowitz NL (2011) Cannabinoid-opioid interaction in chronic pain. Clin Pharmacol Ther 90(6): 844-851.

16. Lynch ME, Clark AJ (2003) Cannabis reduces opioid dose in the treatment of chronic non-cancer pain. Journal of Pain and Symptom Management 25(6): 496498

17. Bryson WC, Morasco BJ, Cotton BP, Thielke SM (2021) Cannabis Use and Nonfatal Opioid Overdose among Patients Enrolled in Methadone Maintenance Treatment. Subst Use Misuse 56(5): 697-703.

18. First L, Douglas W, Habibi B, Singh JR, Sein MT (2020) Cannabis Use and Low-Back Pain: A Systematic Review. Cannabis Cannabinoid Res 5(4) 283-289.
19. Lucas P (2017) Rationale for cannabis-based interventions in the opioid overdose crisis. Harm Reduct J 14(1): 58.

20. Gonen T, Amital H (2020) Cannabis and Cannabinoids in the Treatment of Rheumatic Diseases. Rambam Maimonides Medical J 11(1): e0007.

21. Balneaves LG, Alraja AA (2019) Guarding their practice: a descriptive study of Canadian nursing policies and education related to medical cannabis. Bmc Nurs 18(1): 66.

22. Russo EB (2016) Current Therapeutic Cannabis Controversies and Clinical Trial Design Issues. Front Pharmacol 7: 309.

23. Sarzi-Puttini P, Batticciotto A, Atzeni F, Bazzichi L, Franco MD, et al. (2019) Medical cannabis and cannabinoids in rheumatology: where are we now? Expert Rev Clin Immu 15(10): 1019-1032. 